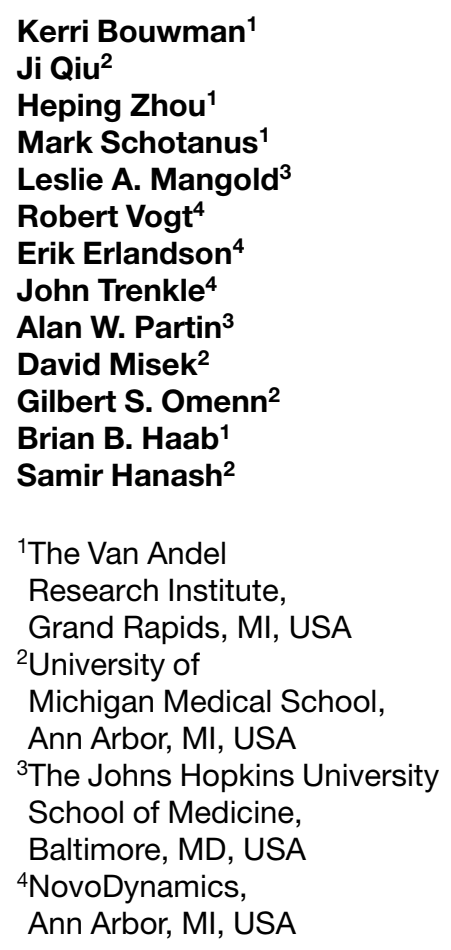

\section{Microarrays of tumor cell derived proteins uncover a distinct pattern of prostate cancer serum immunoreactivity}

\begin{abstract}
The broad characterization of the immune responses elicited by tumors has valuable applications in diagnostics and basic research. We present here the use of microarrays of tumor-derived proteins to profile the antibody repertoire in the sera of prostate cancer patients and controls. Two-dimensional liquid chromatography was used to separate proteins from the prostate cancer cell line LNCaP into 1760 fractions. These fractions were spotted in microarrays on coated microscope slides, and the microarrays were incubated individually with serum samples from 25 men with prostate cancer and 25 male controls. The amount of immunoglobulin bound to each fraction by each serum sample was quantified. Statistical analysis revealed that 38 of the fractions had significantly higher levels of immunoglobulin binding in the prostate cancer samples compared to the controls. Two fractions showed higher binding in the control samples. The significantly higher immunoglobulin reactivity from the prostate cancer samples may reflect a strong immune response to the tumors in the prostate cancer patients. We used multivariate analysis to classify the samples as either prostate cancer or control. In a crossvalidation study, recursive partitioning classified the samples with $84 \%$ accuracy. A decision tree with two levels of partitioning classified the samples with $98 \%$ accuracy. Additional studies will allow further characterization of tumor antigens in prostate cancer and their significance for diagnosis. These results suggest that microarrays of fractionated proteins could be a powerful tool for tumor antigen discovery and cancer diagnosis.
\end{abstract}

Keywords: Protein microarrays / Tumor antigens / Two-dimensional chromatography PRO 0611

\section{Introduction}

Protein microarrays allow high-throughput investigations of protein interactions for a variety of applications. Early feasibility studies showed the detection of specific interactions with purified recombinant proteins spotted onto derivatized glass slides [1] and nitrocellulose [2]. Microarrays of peptides have been used to study the immunoglobulin repertoires in sera of patients with autoimmune diseases [3, 4]. High-throughput protein expression and purification has been used to produce many different types of proteins for microarrays, using bacterial libraries of human cDNA clones that were induced to express protein and arrayed onto membranes [5]. In another demonstration, proteins from the entire yeast proteome were expressed, purified, spotted onto arrays and probed to identify specific protein-protein interactions [6]. The

Correspondence: Dr. Brian B. Haab, The Van Andel Research Institute, 333 Bostwick NE, Grand Rapids, MI 49503, USA

E-mail: brian-haab@vai.org

Fax: +1-616-234-5269

Abbreviation: PSA, prostate specific antigen acquisition of a sufficient variety and quantity of various proteins for microarrays has been a challenge that highthroughput protein expression has in part addressed.

A promising alternative method of producing proteins for microarrays is multidimensional liquid-based separations of proteins from nonrecombinant sources such as human cells and tissues. Multiple modes of separation in succession (for example ion-exchange chromatography followed by reverse phase) yield greater resolving power than single modes of separation, and liquid phase methods allow convenient fraction collection. As previously suggested [7], protein fractions separated by liquid chromatography (LC) and spotted onto microarrays could be used for the parallel interrogation of thousands of proteins. The proteins loaded onto the columns would be taken from their native states, in which modifications and alterations to the proteins are present. In contrast, proteins expressed in foreign systems, such as in bacterial or insect cells, may not have correct post-translational modifications.

An important application of protein microarrays is the study of immune responses in cancer [7, 8]. Many human tumors elicit immune responses to mutant or other- 
wise aberrantly expressed proteins. P53 autoantibodies are common in the sera of patients with multiple cancer types, such as breast [9], colon [10] and prostate [11] cancers. A study of serum antibodies to seven common tumorassociated antigens found that over half of the cancer patients studied showed reactivity to at least one of the antigens [11]. Large-scale profiling of immunoglobulin reactivity in the sera of lung cancer patients uncovered a significant occurrence of antibodies to the proteins annexin I and II [12]. Prostate tumors are often immunogenic for the proteins PSA and HER-2/neu [13], and men with metastatic prostate cancer often produce antibodies against the secretory granules of the prostatic epithelium [14]. The measurement of autoantibodies could have value in the diagnosis of prostate cancer. In addition, a characterization of the immune response in cancer patients could contribute to the understanding of molecular alterations in tumors and the host interaction with the tumors.

We applied our protein microarray of natural proteins approach to study immunogenicity in prostate cancer patients and to determine if distinct patterns of immunoreactivity associated with the disease may be uncovered. We used the LNCaP cell line to produce proteins that were fractionated by IEF and reverse phase chromatography. LNCaP cells retain many of the characteristics of prostate cells and are a good model of androgen-dependent prostate cancer. Microarrays spotted with the chromatography fractions from the $\mathrm{LNCaP}$ proteins were used to characterize the immune recognition of prostate cancer proteins in sera. We investigated whether certain fractions exhibit antibody binding that is associated with prostate cancer and whether the serum samples could be classified based on their patterns of immune reactivity.

\section{Materials and methods}

\subsection{Serum samples}

Serum samples were collected as part of a HIPAA compliant, IRB approved, Early Detection Research Network (EDRN) sponsored protocol at the Johns Hopkins Medical Institution. Samples from 25 men with prostate cancer were collected prior to a prostatic biopsy or prostatectomy that had been dictated by an elevated prostate specific antigen (PSA) reading ( $>4.0 \mathrm{ng} / \mathrm{mL}$ ) or an abnormal digital rectal examination (DRE). Each patient underwent radical retropubic prostatectomy. The tumors were Gleason grade 6 or 7 and had not infiltrated the lymph nodes or seminal vesicles. Control sera were collected from 25 age-similar men with normal serum PSA levels and a negative DRE as part of a routine community screening program. The samples were stored frozen at $-80^{\circ} \mathrm{C}$ and had been thawed one time prior to use.

\subsection{Cell culture and protein extraction}

The LNCaP cell line (purchased from ATCC, Manassas, VA, USA) was grown in RPMI 1650 medium with $10 \%$ fetal calf serum. We collected protein from 12 P100 culture dishes containing approximately 9.5 million cells per plate, resulting in approximately $50 \mathrm{mg}$ of protein extract. The cells were solubilized in a solution of $6 \mathrm{~m}$ urea, $2 \mathrm{M}$ thiourea, $1.0 \%$ n-octyl b-D-glucopyranoside, $2 \mathrm{~mm}$ dithioerythritol, protease inhibitor cocktail (Roche, Basel, Switzerland), and 2\% carrier ampholytes, $\mathrm{pH} 3.5-10$ (BioRad, Hercules, CA, USA). The protein extract was stored at $-80^{\circ} \mathrm{C}$ until ready for use.

\subsection{Protein fractionation}

The Hanash laboratory has experimented with several procedures to obtain liquid based protein separations in two dimensions that are compatible with the printing of all fractions on microarrays. In this study, we have coupled a preparative-scale Rotofor (Bio-Rad) separation in the first dimension with the use of reverse phase in the second dimension [7]. For the first dimension separation, whole cell protein lysates were diluted to $55 \mathrm{~mL}$ with a running buffer consisting of the lysis buffer and $0.5 \%$ n-octyl $\beta$-Dglucopyranoside. Proteins were separated by IEF for $6 \mathrm{~h}$ at $10^{\circ} \mathrm{C}$. Twenty separate fractions were collected. The protein concentration and $\mathrm{pH}$ of each fraction was measured as previously described [7]. We have ascertained that, when reacted with patient and control sera, aliquots from cancer cell lines spotted after even the first dimension separation exhibited distinct patterns of reactivity with individual sera (data not shown).

The high-performance liquid chromatography (HPLC) reversed phase column R2/10 (Applied Biosystems, Foster City, CA USA) was used for the separation of proteins in the second dimension. Separations were performed at a flow rate of $1.3 \mathrm{~mL} / \mathrm{min}$ using water/acetonitrile gradients (solvent $\mathrm{A}$ : $98 \% \mathrm{H}_{2} \mathrm{O}, 2 \%$ acetonitrile, 0.1\% TFA; solvent $\mathrm{B}$ : $90 \%$ acetonitrile, $10 \% \mathrm{H}_{2} \mathrm{O}, 0.1 \%$ TFA). The gradient profile used was as follows: (0) $95 \%$ solvent $A$ for $2.5 \mathrm{~min}$; (1) 95-75\% A in $2.5 \mathrm{~min}$; (2) 75-35\% A in $40 \mathrm{~min}$; (3) $35 \%$ A for $5 \mathrm{~min}$; (4) $35-15 \%$ A in $5 \mathrm{~min}$; (5) $15-5 \% \mathrm{~A}$ in $5 \mathrm{~min}$. Protein fractions were collected every $30 \mathrm{~s}$ (88 fractions from each of the 20 first dimension fractions) starting $10 \mathrm{~min}$ into the gradient, then immediately frozen at $-80^{\circ} \mathrm{C}$. The fractions were lyophilized under vacuum, resuspended in $35 \mu \mathrm{L}$ of $80 \% \mathrm{PBS} / 20 \%$ glycerol, and frozen at $-80^{\circ} \mathrm{C}$. An estimated average of $3.5 \mu \mathrm{g}$ of protein was collected in each fraction, resulting in a $100 \mu \mathrm{g} / \mathrm{mL}$ average protein concentration in the resuspended samples. We have also ascertained that when reacted with patient and control sera, aliquots from cancer cell lines 
spotted after separation in one dimension by reverse phase HPLC exhibited distinct patterns of reactivity with individual sera (data not shown).

\subsection{Protein microarrays}

The resuspended LNCaP fractions were transferred to polypropylene 384-well plates. The following controls were added to empty wells of the 384-well plates: (1) $50 \mu \mathrm{g} / \mathrm{mL}$ biotinylated anti-Ig (Amersham Biosciences, Piscataway, NJ, USA); (2) anti-IgG (biotinylated and not), anti-IgM, and anti-albumin (Bethyl, Montgomery, TX, USA); (3) prostate specific antigen and tetanus toxoid (Calbiochem, La Jolla, CA, USA); (4) anti-DNP (Biotrend, Cologne, Germany) and anti-Flag (Sigma, St. Louis, MO, USA); and (5) biotinylated and digoxigenin conjugated forms of Flag-labeled bacterial alkaline phosphatase (Sigma) and DNP-labeled BSA. The plates were stored at $-80^{\circ} \mathrm{C}$.

The protein solutions were printed in microarrays on the surfaces of nitrocellulose-coated microscope slides (Schleicher \& Schuell, Keene, NH, USA) using a custombuilt microarraying robot. The microarrays for this study were printed in one batch. Each solution was printed in duplicate in adjacent spots. The slides were stored sealed at $4^{\circ} \mathrm{C}$. The printed slides were rinsed for $30 \mathrm{~s}$ in $1 \times \mathrm{PBS}$, $0.5 \%$ Tween-20 (PBST0.5), then washed with gentle rocking at room temperature for $3 \mathrm{~min}$ and $30 \mathrm{~min}$ in fresh changes of PBST0.5. The slides were blocked for one hour in $1 \times$ PBS, $0.1 \%$ Tween-20 (PBST0.1) at room temperature with gentle shaking, followed by a brief rinse in PBST0.5 and drying by centrifugation. The array boundaries on each slide were circumscribed with a hydrophobic marker (PAP pen; Polysciences, Warrington, PA, USA) about 3-4 mm outside the edges of the arrays. The marker lines were allowed to dry completely before incubation.

Each array was incubated with serum that had been diluted 50-fold in PBST0.1 and spiked with biotinylated/Flaglabeled bacterial alkaline phosphatase (BAP) fusion protein and biotinylated/DNA-labeled BSA (for normalization). The final concentrations of the Flag and DNP labeled controls were $0.5 \mu \mathrm{g} / \mathrm{mL}$ and $1 \mu \mathrm{g} / \mathrm{mL}$, respectively. Three hundred microliters of each serum solution was incubated on each array for $1.5 \mathrm{~h}$ at room temperature with gentle shaking. The arrays were rinsed for $30 \mathrm{~s}$ in PBST0.1 and washed with gentle rocking at room temperature $3 \times 5$ min each in fresh changes of PBST0.1. Slides were then dried by centrifugation.

Five $\mathrm{mg} / \mathrm{mL}$ of biotinylated anti-lg (Amersham Biosciences) in 3\% nonfat milk/PBST0.1 was incubated on the arrays for one hour at room temperature with gentle shaking. Slides were rinsed and washed as above and dried by centrifugation. A final incubation of $10 \mu \mathrm{g} / \mathrm{mL}$ streptavidin-phycoery- thrin (Amersham Biosciences) in 3\% nonfat milk/PBST0.1 was carried out for one hour at room temperature with gentle shaking. The slides were rinsed, washed and dried as above and stored at $4^{\circ} \mathrm{C}$ until scanned.

\subsection{Analysis}

The microarrays were scanned for fluorescence (ScanArray; PerkinElmer Life Sciences, Boston, MA, USA) using consistent detector gain and laser power settings (excitation at $532 \mathrm{~nm}$ ) for all arrays. The program GenePix 4.0 (Axon Laboratories, Union City, CA, USA) was used to calculate the median background-subtracted pixel intensity of each spot and to assign user-supplied identification tags to each spot. The identification tags were based on each fraction's location in the 96-well plates that were collected after fractionation (e.g., P2B10 refers to plate 2, well $B 10)$. An intensity threshold for each spot was calculated by the formula $3^{*} B^{*} C V_{b}$, where $B$ is each spot's median local background and $\mathrm{CV}_{\mathrm{b}}$ is the average coefficient of variation (SD divided by the average) of all the local backgrounds on the array. (This threshold is similar to the SD of the local background but minimizes the effects from spikes in the local backgrounds.) Duplicate spots with background-subtracted intensities surpassing the threshold were averaged, and spots not surpassing the threshold in either of the duplicates were given a value of zero. Normalization could be performed using data from the anti-DNP and anti-Flag spots on the array, which bound the control spikes that had equivalent concentrations in all the samples. Normalization had a near negligible effect on the overall trends in the data and was not used in the preparation of the data for subsequent analyses.

The program CIT [15] was used to identify fractions that statistically discriminated the patient groups. CIT performed a permutation $t$-test using 100 permutations and a maximum $p$-value of 0.01 . Hierarchical clustering and the visualization of clusters were performed using the programs Cluster and Treeview (http://rana.Ibl.gov/Eisen Software.htm). Data were median centered by genes prior to average-linkage clustering. Recursive partitioning to classify the samples was performed using the program ArborPharm $^{\mathrm{TM}}$ (NovoDynamics, Ann Arbor, MI, USA).

\section{Results}

1760 fractions were collected from the 2-D liquid chromatography separation of proteins from the prostate cancer cell line LNCaP. The fractions and control proteins were spotted in microarrays on nitrocellulose-coated microscope slides, and each of 25 sera from men with prostate cancer and 25 sera from age-similar healthy men were incubated individually, each on a separate microarray. 
Immunoglobulins from the sera that bound to spotted fractions were detected by incubating the microarrays with biotinylated anti-human Ig and a phycoerythrin-streptavidin conjugate, followed by scanning the microarrays for fluorescence. Representative scanned images (Figs. 1A and $1 \mathrm{~B}$ ) from both the cancer and control sera show a consistent level of background signal and multiple spots with fluorescence well above the background. Each fraction was spotted in duplicate adjacent spots, and consistent pairs of spots are observed in both images. When anti-lg and streptavidin-phycoerythrin were incubated on the arrays without serum, only the biotinylated and control proteins showed strong signal (data not shown).

The median net fluorescence intensity (after subtraction of the local background signal) at each spot was calculated, and the signal from duplicate spots was averaged.
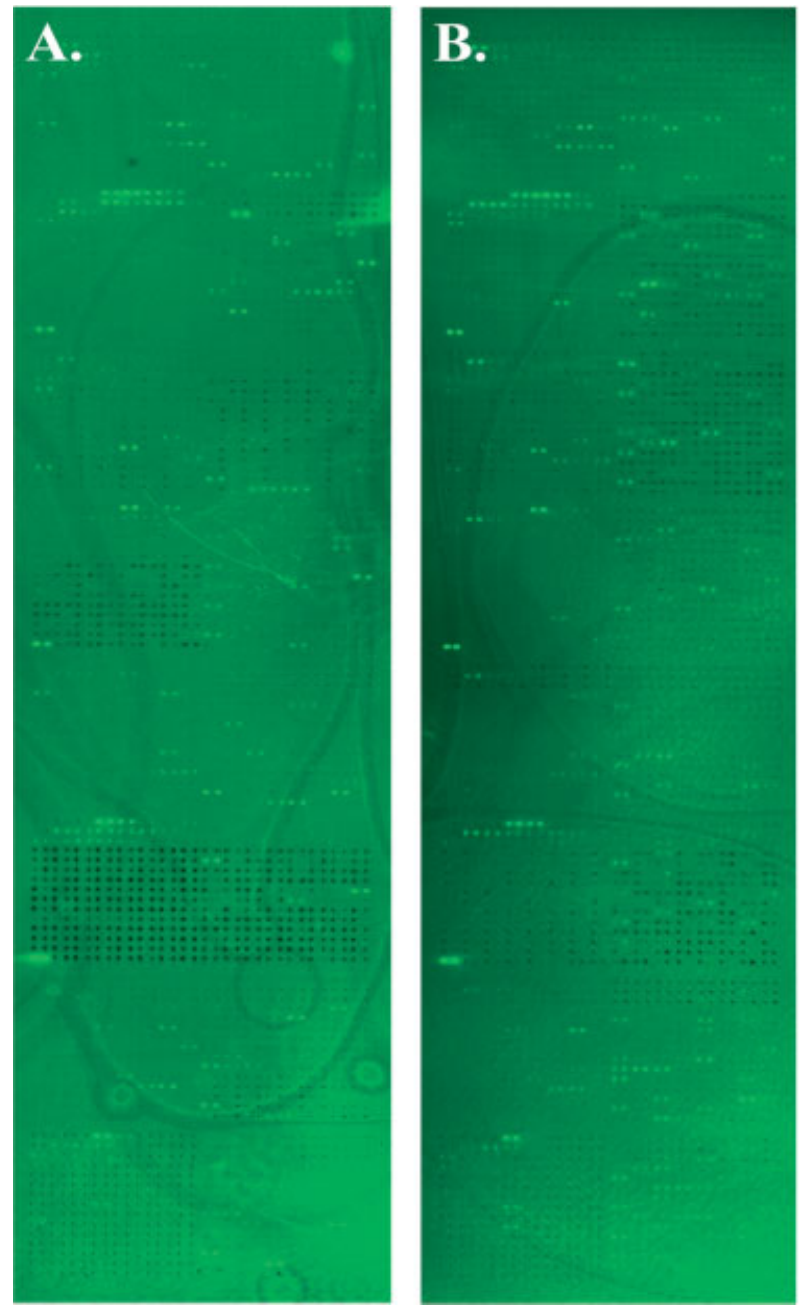

Figure 1. Representative scanned images of microarrays that had been incubated with serum from $(A)$ a man with prostate cancer and (B) a control. The array dimensions were $9 \times 36 \mathrm{~mm}$. Each fraction or protein was spotted in duplicate in adjacent spots.
Spots that had net signal below a statistically-derived intensity threshold (see Section 2.5) in both replicates were given a value of zero. An average of 149 fractions per array (including the 15 control proteins) showed measurable signal above background, and no statistical difference between the cancer samples and the controls was observed in the total number of reactive fractions per array.

Hierarchical clustering allows visualization of trends in data from multiple microarray experiments [16]. Data from all 50 microarray experiments were grouped and clustered by similarity in intensity pattern (Fig. 2A). The left 25 columns of Fig. 2A represent data from the cancer samples, and the right 25 columns represent data from the control samples. Some fractions showed reactivity in all the samples, as indicated by rows that are filled over all 50 columns. Most of these rows represent data from control spots that bound antibodies in every sample or that bound the anti-Ig detection antibody (e.g., tetanus toxoid or biotinylated BSA). Other fractions showed minimal or scattered reactivity. Some patient samples showed significantly more overall reactivity than others, as shown by the vertical stripes in the cluster that indicated binding by a large number of fractions.

A comparison of the relative levels of antibody binding from the prostate cancer and control sera was investigated. A permutation $t$-test applied to the entire data set identified 40 fractions at a $99 \%$ confidence level that showed significantly different levels of reactivity between the two sample groups. Thirty-eight of the discriminating fractions had higher reactivity in the prostate cancer sera, and two fractions had higher reactivity in the control sera. None of the control proteins showed data that was significantly different between the groups. The discriminating fractions were randomly distributed throughout the array, indicating a lack of systematic bias from array location. All of the serum samples had measurable reactivity to at least some of the 40 fractions; the prostate cancer and control samples reacted with averages of 27 and 19 fractions, respectively. Many of the reactive fractions contain the same proteins, since they were collected in consecutive fractions. Further analysis of the fractions with mass spectrometry will be required to clarify the number of immunogenic proteins.

The data from the discriminating fractions were visualized using hierarchical clustering (Fig. 2B). Significantly higher reactivity in the prostate cancer sera is indicated by the generally red-colored data points in the prostate cancer samples and the generally green-colored data points in the control samples. The two fractions with higher reactivity in the control samples are in the bottom rows. The fractions P20B10 and P8B8 (highlighted in the cluster) had 


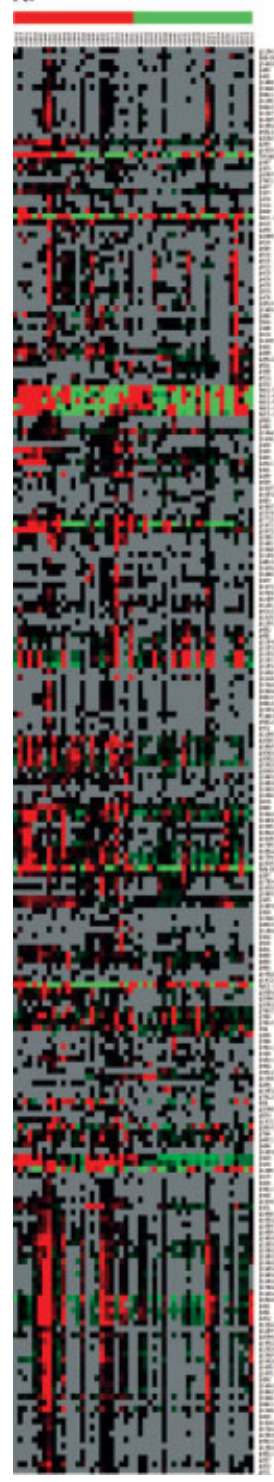

B.

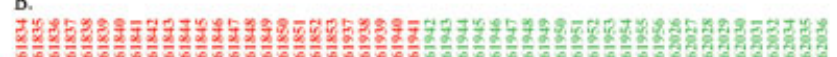

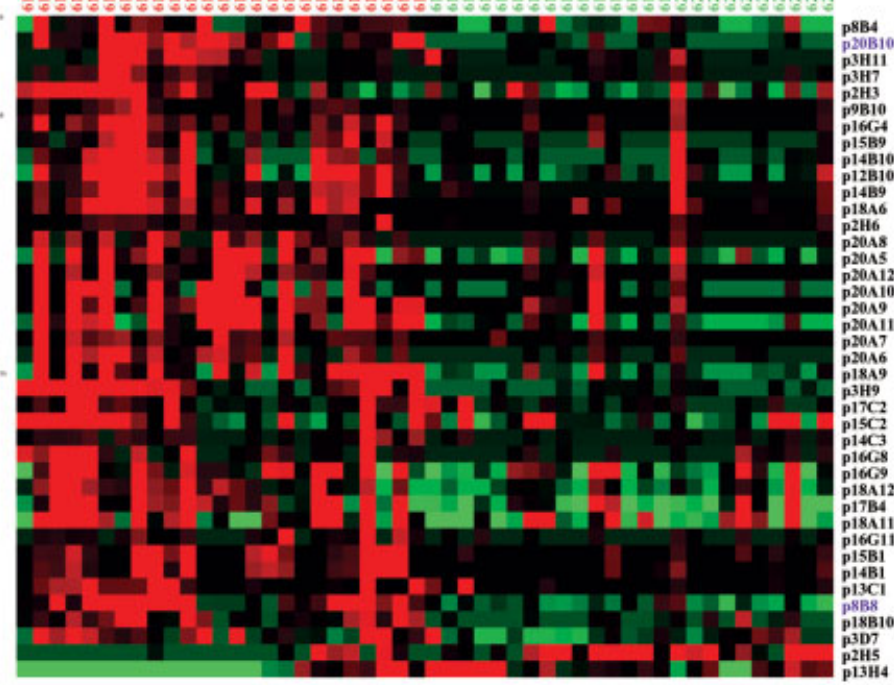

C.

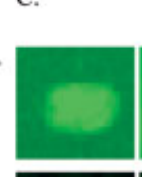

Cancer

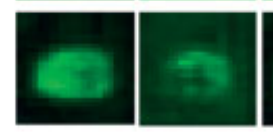

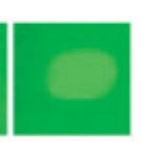

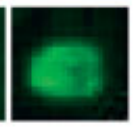

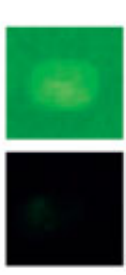

Controls
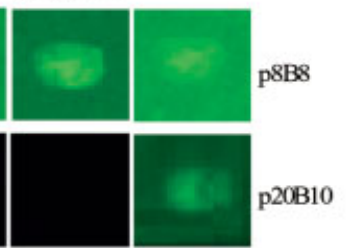

Figure 2. (A) Hierarchical clustering of data from all 50 arrays. Data were median centered (by rows), filtered for rows that contained data in at least $20 \%$ of the samples, and clustered by rows (the column order was fixed). Each column represents data from one microarray, and each row represents data from one fraction. The left $25 \mathrm{col}-$ umns (indicated by the red bar) are data from arrays incubated with prostate cancer sera, and the right 25 columns (indicated by the green bar) are data from arrays incubated with control sera. The color of each square indicates the signal intensity relative to the other data in a given row. Red indicates high intensity, green indicates low intensity, and gray is no data (no signal detected). (B) Hierarchical clustering of fractions that significantly discriminate the groups. Data were treated and clustered as described above, except that blank data were given a value of zero. Green data points indicate low intensity (relative to other data points in a given row), black indicates median values, and red indicates high intensity. The labels to the rows containing data from the fractions P8B8 and P20B10 are highlighted. (C) Detailed view of several spots from fractions P8B8 and P20B10 after incubation with either cancer sera (left) or control sera (right). Each array was scanned at equivalent scanner settings, and each image was created with equivalent brightness and contrast settings.

the highest statistical significance of discrimination. An examination of representative spots from these two fractions in samples from both groups of patients (Fig. 2C) shows the higher level of binding from the prostate cancer sera. A more quantitative view of the signal intensities from these two fractions shows the differences in antibody binding between the prostate cancer and control samples (Fig. 3). All 25 of the prostate cancer sera and 10 of the normal sera showed reactivity to the P20B10 fraction, with average signal intensities of 919 and 104, respectively. Twenty-five of the prostate cancer sera and 23 of the normal sera had some measurable signal at fraction P8B8, but the average signal intensity from the prostate cancer sera was higher: 1257 versus 392 in the control sera.

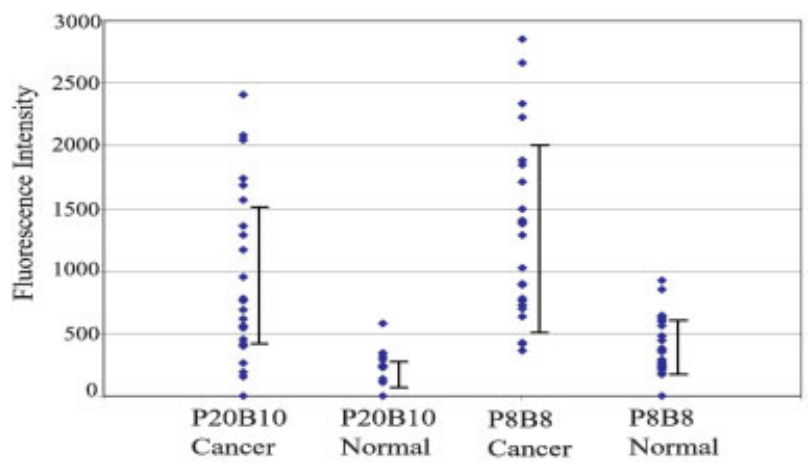

Figure 3. Signal intensities measured at fractions P8B8 and P20B10 in the cancer sera and control sera. The error bars indicate two standard deviations above and below the mean value. 
We next explored the potential for the diagnostic classification of serum samples based on measurements of cancer-associated immunoreactivity. An advantage of the highly-parallel measurements enabled by protein microarrays is the ability to combine multiple measurements to potentially improve the diagnostic accuracy over individual measurements. Methods to combine multiple measurements include discriminant analysis, logistic regression, neural networks, and decision trees. We evaluated the use of recursive partitioning (also known as decision trees) and ensemble techniques [17] to classify the serum samples into either the cancer group or the control group based on the intensity profiles of the fractions. Recursive partitioning is a supervised learning technique that attempts to construct tree structured models that can then be used to classify unknown samples. Some of the strengths of recursive partitioning are that it can handle large numbers of descriptors (in this case, fractions), and it can assess the relative importance of the descriptors for the task of classification.

We first used cross-validation techniques to determine if the dataset could be accurately classified using recursive partitioning. We used a "leave-one-out" method for crossvalidation, whereby one sample is left out for independent testing and the other samples are used as a training set to define the best decision tree. The decision tree is then used to classify the sample that had been left out. That process is repeated for all samples. Using this analysis we classified samples into their correct groups with $86 \%$ accuracy. This result indicates that the decision trees should have considerable accuracy when tested against independent data. We then constructed a tree based on all the samples (Fig. 4). The p20B10 fraction was powerful for initially splitting the data at the first layer of the tree; this fraction correctly classified $21 / 25$ cancer samples (84\% sensitivity) and $24 / 25$ control samples (96\% specificity). The second layer used the fractions P3D2 and P14E8 to further split the data locally. After the second layer of the tree, all cancer samples were correctly classified and only one normal sample was incorrectly classified as a cancer sample.

To determine which fractions provide the most information for classification, all the fractions were tested for their ability to classify the samples by recursive partitioning using only that fraction at every level of the decision tree. Fifteen fractions classified the samples with an accuracy of $80 \%$ or greater (Table 1). There was substantial agreement between the fractions identified by recursive parti-

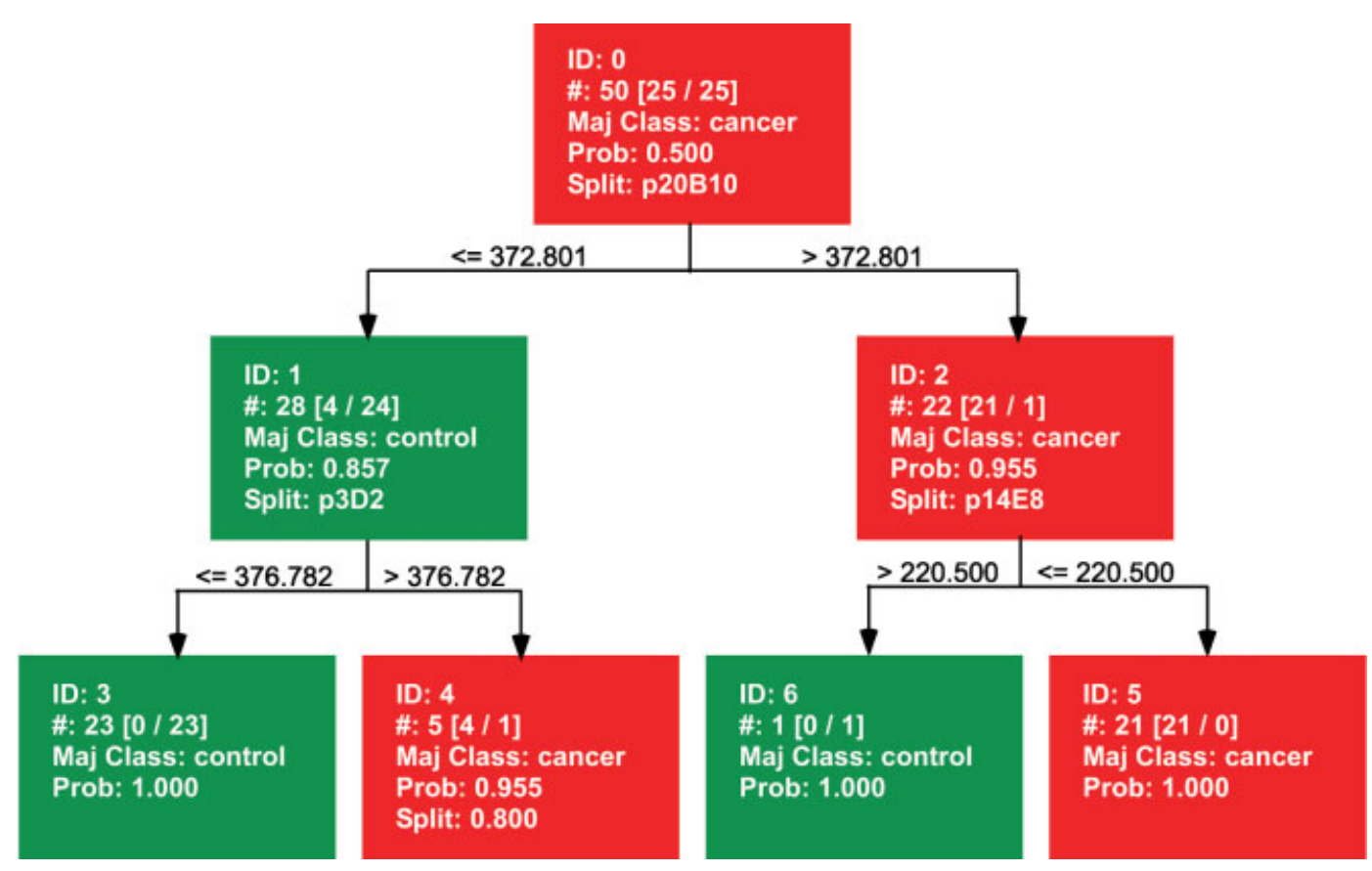

Figure 4. A decision tree showing that the 50-sample dataset can be discriminated with $98 \%$ accuracy using measurements from $\mathrm{P} 20 \mathrm{~B} 10$ in the first level and measurements from P3D2 and P14E8 in the second level. A red box indicates classification as cancer, and a green box indicates classification as control. The actual number of cancer/control samples in each group is given in each box. The protein fraction used to make a distinction is indicated after the term Split, and the values below each split are the thresholds used in the split. 
Table 1. Fractions that classified the 50 samples with greater than or equal to $80 \%$ accuracy by recursive partitioning. For each fraction, recursive partitioning was used to classify the samples using only that fraction in all levels of the decision tree.

\begin{tabular}{ll}
\hline Fraction & Accuracy $(\%)$ \\
\hline p20B10 & 94 \\
p8B8 & 92 \\
p3H9 & 88 \\
p18A9 & 84 \\
p20A9 & 82 \\
p18B10 & 82 \\
p20A5 & 82 \\
p2H4 & 82 \\
p3H5 & 82 \\
p7B10 & 80 \\
p14B1 & 80 \\
p20A11 & 80 \\
p6B1 & 80 \\
p2H5 & 80 \\
p4D9 & 80 \\
\hline
\end{tabular}

tioning and those identified by the permutation $t$-test. Of the 15 fractions in Table 1, the top seven (P20B10 through P20A5) and three others (P14B1, P20A11 and $\mathrm{P} 2 \mathrm{H} 5)$ were among the 40 fractions identified by the permutation $t$-test. The two top fractions (P20B10 and P8B8) were also the top two identified by the permutation $t$-test.

\section{Discussion}

A primary goal of this study was to explore the usefulness of microarrays of protein fractions for the identification of cancer-specific immunoreactivity. The effectiveness of the method was shown in several ways. The strong fluorescence signal above background from many of the fractions clearly indicated that the method has sufficient sensitivity to detect the binding of specific antibodies. The strong signal from each of the control proteins also confirmed that the method worked properly. A permutation $t$-test identified 40 fractions with generally higher binding from the prostate cancer samples than from the normal samples and two fractions with generally higher binding from the normal samples. None of the control proteins had significantly different measurements between the sample groups.

The significant nonrandom predominance of higher binding strengths from the prostate cancer samples could indicate a high immune response to the prostatic tumor in the prostate cancer patients. Multiple proteins seem to have stimulated an immune response in the prostate cancer patients. Many of the discriminating fractions likely share the same protein responsible for the antibody binding because they had been consecutively collected after the chromatography. For example, fractions P20A5 through to P20A20 were consecutively collected and all have similar signals over all the samples. Some of the discriminating fractions showed low-level binding in all the normal samples. Low signal strengths from all the samples could indicate a particularly 'sticky' fraction, to which many proteins nonspecifically bind. For this reason further investigation into the immunogenicity of the proteins in the fractions will be required. The identification of the particular proteins in the fractions by MS will aid the further characterization and validation of the immunogenicity of the proteins and investigation into the causes of the immungenicity. It would be valuable to know whether an immune response to the protein is induced by abnormally abundant presentation to the immune system, mutations, or aberrant protein modifications.

A second goal of the study was to explore the potential for identification of prostate cancer patients through multivariate analysis. The use of the measurements to accurately classify the samples as either from prostate cancer or control patients was tested using recursive partitioning. Recursive partitioning classified the samples with $90 \%$ accuracy (5 misclassifications out of 50 samples) at the first level of partitioning using the fraction P20B10. The accuracy improved to $98 \%$ accuracy (1 misclassification out of 50) after the second level of partitioning using fractions P3D2 and P14E8. The improvement using multiple tree levels shows the potential benefit from using multiple measurements to classify samples. Recursive partitioning also was used to identify fractions that individually classified the data with high accuracy (Table 1). Most of the fractions in Table 1 were also identified by the permutation $t$-test, further confirming their statistical significance. Multivariate methods require high sample numbers for validation, and therefore further experiments using these fractions to probe larger sample sets will be required. Expanded studies also will include sera from patients with benign prostatic diseases, localized and metastatic cancer, androgen responsive and androgen independent cancer, and other cancers.

Experiments from multiple different batches of microarrays also will be important to account for potential systematic variability in a particular batch. The signal strength at each fraction is influenced by the density of the spotted fraction, which can be highly variable using microarrays spotted with contact pin printers. Therefore in addition to performing more randomized experiments, 
a validation study could be performed using microarrays prepared using noncontact piezoelectric spotters, which deposit very consistent volumes onto microarrays.

\section{Concluding remarks}

These promising results suggest the possibility of using patterns of immune recognition as a diagnostic test. A previous example of the use of serum protein patterns for prostate cancer diagnostics employed multivariate analysis of surface enhanced laser desorption/ionization mass spectrometry data [18]. A related approach using measurements of the immune system repertoire could likewise prove useful. A great need exists for supplementation of the PSA test and other clinical indicators in the management of prostate cancer patients, particularly for the better discrimination of benign from malignant disease, better prediction of treatment success, and earlier identification of fast-growing, invasive or metastatic cancers. The measurement of serum antibody reactivity to multiple prostate cancer antigens could eventually be valuable for these objectives. Considering the widespread observations of tumor antigenicity in other types of cancers, microarrays of fractionated proteins may be a powerful general tool for the broad screening of tumorassociated antigens.

The work was supported by grant MEDC 238 from the Michigan Life Sciences Corridor for the Proteomics Alliance for Cancer.

Received May 20, 2003

\section{References}

[1] MacBeath, G., Schreiber, S. L., Science 2000, 289, 17601763.

[2] Ge, H., Nucleic Acids Res. 2000, 28, e3.

[3] Robinson, W. H., DiGennaro, C., Hueber, W., Haab, B. B. et al., Nat. Med. 2002, 8, 295-301.

[4] Joos, T. O., Schrenk, M., Hopfl, P., Kroger, K. et al., Electrophoresis 2000, 21, 2641-2650.

[5] Lueking, A., Horn, M., Eickhoff, H., Buessow, K. et al., Anal. Biochem. 1999, 270, 103-111.

[6] Zhu, H., Bilgin, M., Bangham, R., Hall, D. et al., Science 2001, 293, 2101-2105.

[7] Madoz-Gurpide, J., Wang, H., Misek, D. E., Brichory, F. et al., Proteomics 2001, 1, 1279-1287.

[8] Yan, F., Sreekumar, A., Laxman, B., Chinnaiyan, A. M. et al., Proteomics 2003, 3, 1228-1235.

[9] Regele, S., Vogl, F. D., Kohler, T., Kreienberg, R. et al., Anticancer Res 2003, 23, 761-764.

[10] Hallak, R., Mueller, J., Lotter, O., Gansauge, S. et al., Int. J. Oncol. 1998, 12, 785-791.

[11] Zhang, J. Y., Casiano, C. A., Peng, X. X., Koziol, J. A. et al., Cancer Epidemiol Biomarkers Prev. 2003, 12, 136-143.

[12] Brichory, F. M., Misek, D. E., Yim, A. M., Krause, M. C. et al., Proc. Natl. Acad. Sci. USA 2001, 98, 9824-9829.

[13] McNeel, D. G., Nguyen, L. D., Storer, B. E., Vessella, R. et al., J. Urol. 2000, 164, 1825-1829.

[14] Nilsson, B. O., Carlsson, L., Larsson, A., Ronquist, G., Ups. J. Med. Sci. 2001, 106, 43-49.

[15] Rhodes, D. R., Miller, J. D., Haab, B. B., Furge, K. A., Bioinformatics 2002, 18, 205-206.

[16] Eisen, M. B., Spellman, P. T., Brown, P. O., Botstein, D., Proc. Natl. Acad. Sci. USA 1998, 95, 14863-14868.

[17] Breiman, L., Friedman, J. H., Olshen, R. A., Stone, C. J., Classification and Regression Trees, Wadsworth \& Brooks/ Cole Advanced Books \& Software, Pacific Grove, CA 1984.

[18] Petricoin, E. F., ${ }^{\text {rd }}$, Ornstein, D. K., Paweletz, C. P., Ardekani, A. et al., J. Natl. Cancer Inst. 2002, 94, 1576-1578. 